\title{
Esclavitud Urbana y Tipologias Habitacionales en Montevideo
}

Carlos Altezor*

En el tema de la historia de la esclavitud negra en el Uruguay, desglosamos una visión histórico-arquitectónica sobre particulares modos de alojamento de esclavos negros en Montevideo bajo la dominación española, en la casa patriarcal de patios; durante la época post-colonial, en viviendas colectivas para las clases bajas de la sociedad republicana.

En el Montevideo colonial, el esclavo negro sirve a sus amos principalmente en la residencia urbana del español enriquecido. Con el advenimiento de la República en 1830 y el proceso de liberación de la esclavitud, el afincamiento del liberado y su familia se realiza en determinadas áreas de la ciudad, donde la casa de inquilinato y el típico programa arquitectónico del conventillo son los lugares de habitación destinados para él, junto a otros sectores sociales desposeídos. La cultura negra de raíces africanas, modelada por circunstancias históricas y geográficas, supo permanecer en el tiempo, apegada a aquellos ámbitos urbanos que la ciudad reconoce que la expresan, como son principalmente los barrios Sur y Palermo. Aunque el autoritarismo, la especulación y la ignorancia atentan a menudo contra ella, destruyendo inegualables monumentos de su patrimonio social y cultural y del país todo.

* Em co-autoria com a arqa Mara Moya. Colaboraçăo da arqa Maria Julia Gomes. 
Reseña sobre la introducción y desarrollo de la esclavitud en la Banda Oriental

El territorio de la Banda Oriental experimenta un tardío proceso fundacional y posterior colonización con respecto al resto de la América Española. Factores geopolíticos - presencia de Portugal en Colonia del Sacramento desde 1680 - y de defensa de la soberanía española en este territorio posibilitan su ocupación a partir de la fundación de Montevideo por Zabala en 1724. En el marco histórico de escaso poblamiento y carencia de explotación económica que requiera mano de obra en cantidades importantes, es explicable la particular característica que tuvo la presencia del esclavo negro en la época colonial; carece de importancia productiva, en términos relativos a otras regiones del imperio español y generalmente su posesión tiene caracter suntuario y de ostentación para el poblador español.

Nuestra historiografía reconoce tres momentos en la introducción de esclavos en la colonia. El primero tiene que ver con la fundación de la Colonia del Sacramento y la presencia de esclavos en ella y también por el accionar del comercio negrero por medio del contrabando, desde territorios limitrofes con las posesiones portuguesas y por mar, con el pretexto de "arribada forzosa" de navíos extranjeros con carga diversa y esclavos, que el Cabildo autoriza a desembarcar y mercar finalmente en Montevideo. Un segundo período lo señala la fecha de 1743, a partir de la cual comienzan a llegar a puerto los primeros barcos con cargamento de esclavos, autorizados por el regimen de Asientos. El tercer momento está dado por el establecimiento del regimen de libre trafico en el comercio negrero desde 1789 y por Real Cédula, el 28 de febrero de 1791, Montevideo es declarado único puerto de entrada de esclavos para la zona sur del continente, incluidos Chile y Perú. En este último período se registran los mayores ingresos de población negra, resultando una existencia de un $22 \%$ de población esclava en la ciudad, según el censo del Cabildo en 1803. En datos de 1829, el esclavo en el territorio de lo que es hoy el Uruguay sumaba 2.489 , sobre una población total de 74.000 , sin contar los libertos. En el correr del siglo XIX el peso relativo de la población negra disminuye paulatinamente por los contingentes inmigratorios europeos que comienzan a llegar al país y particularmente por haber formado la vanguardia de los ejércitos que se enfrentaron en las frecuentes guerras civiles que se sucedieron en el Uruguay. 


\section{El proceso abolicionista}

Cuarenta años dura el proceso abolicionista de la esclavitud en el país. Desde la resolución de la Asamblea General de las Provincias Unidas de 1813 que establece la libertad de vientres - ratificada en 1825 en la Asamblea de la Florida - pasando por la ley del 13 de octubre de 1835 prohibiendo el tráfico negrero, hasta la del 16 de junio de 1837 acordando la libertad a los esclavos que ingresan al país y según lo ya dispuesto por la Constitución Nacional de 1830. Sin embargo, la introducción de esclavos mediante el contrabando permanece hasta 1839, cuando Uruguay, Argentina e Inglaterra, firman un tratado prohibiendo el tráfico negrero y acordando la inspección marítima para controlarlo. El proceso culmina con la ley del 2 de mayo de 1853 , sancionando la libertad para menores negros y la del 23 de julio del mismo año, declarando la trata de esclavos como acto de piratería.

\section{Un primer alojamiento para} esclavos negros en Montevideo

A comienzos del siglo XVIII llega una partida de esclavos a Buenos Aires y simultáneamente se propaga una epidemia de viruela. Era comín que los negros llegaran a su lugar de arribo luego de deficientes e inhumanas condiciones de trato y viaje, con enfermedades de todo tipo, origen de frecuentes epidemias según se decia en la época. El Cabildo bonaerense comienza a tomar prevenciones frente a los temores de la población. Cuando en 1705 se anuncia el arribo de otro cargamento, se genera una inquietud y el gobernador dicta una resolución para que los buques que condujesen enfermos o convalescientes se sometieran a cuarentena al otro lado del río, eligiéndose para ésto a Montevideo, a cuyo puerto se envían médicos para la visita. Cuando se conoce en 1787 la llegada del primer barco de la Compañía de Filipinas - compañía inglesa que había celebrado con España un tratado de asiento - el Cabildo de Montevideo dispuso que se conminara al apoderado de dicha compañía a "que disponga de habitación bastante para los negros que se esperan y demás que sucesivamete vendrán a este puerto, en la boca del arroyo Miguelete hacia la parte del Cerro, que es el paraje que está a la costa del mar y se nombra Jesús María, distante de esta Ciudad tres cuartos de legua: en cuyo puerto deberán pemanecer como el más cómodo para ellos 
mismos y sin riesgo alguno para el publico". De esta manera, la compañía se hizo cargo de la costrucción de un alojamiento en el paraje indicado, que se le conoció como "Caserío de los negros", de caracter transitorio y colectivo en la zona de Propios de la ciudad fortificada de Montevideo (Fig. 1). Es muy poco lo que se conoce de este alojamiento y una de las pocas referencias del mismo es la que corresponde a Isidoro de María, quien lo describe diciendo que "ocupaba una manzana de terreno, bajo muro, teniendo en el centro cinco piezas edificadas, con grandes almacenes, cocina, techos de teja, etc.". Cuando en 1789 fue declarada la libertad de tráfico, el caserío siguió siendo utilizado para "depósito" de esclavos, sirviendo posteriormente para alojamiento de tropas y otros usos, hasta que hacia fines del pasado siglo, la extensión de la ciudad borró todo vestigio de este sitio para cuarentena.

\section{El esclavo negro en la}

\section{casa patriarcal montevideana}

En el período colonial, el esclavo no parece haber sido mayormente destinado al trabajo agropecuario en las estancias cercanas a Montevideo. Se le ocupa sin duda, en la actividad saladeril y en el pequeño cultivo de susistencia, pero sus funciones son principalmente de servidores domésticos. Su posesión y uso se vincula también fuertemente a factores de prestigio social, más que a razones economicas en el Montevideo colonial. De particular importancia resulta la presencia de esclavos en la residencia urbana de españoles o extranjeros adinerados de la ciudad. Comerciantes dedicados a la exportación e importación, hacendados, asentistas y funcionarios enriquecidos a fines del periodo de la dominación española, sobretodo gracias al auge comercial que toma Montevideo y su puerto después de la Pragmática de Libre Comercio, cuentan con un número importante de esclavos a su servicio en las viviendas que a la vez que funcionan como comercio o almacén, constituyen su residencia familiar.

A partir del decenio de los ochenta, se conforma la tipología de vivienda colonial que denominamos casa patio, de caracter patriarcal. Vivienda organizada en base a patios centrales donde se vuelcan las habitaciones y locales. Un patio principal y delatero organiza la tarea comercial y la actividad privada de la família, que se desarrolla en planta 


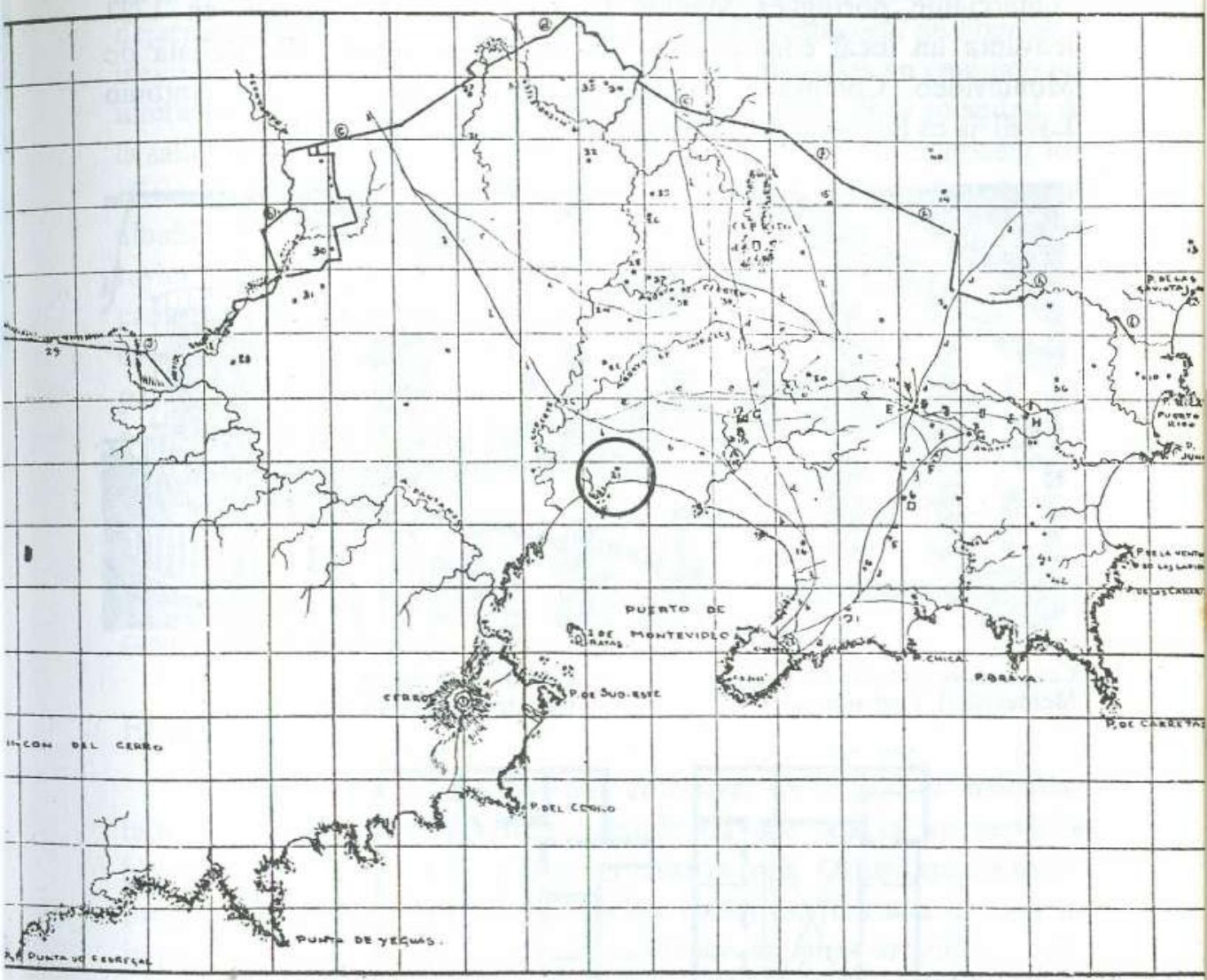

FIG. 1 - Croquis realizado por el Arquitecto Perez Montero en 1940, según plano del Coronel de Ingenieros Desiderio Cony en 1826, ubicando el "Caserío de los negros". 
alta cuando la vivienda es de dos plantas. Un patio posterior nuclea las funciones de servicio, con cocinas, cocheras y particularmente locales para los esclavos de servicio. Ejemplo de ésto, es la casa del rico comerciante portugués Manuel Cipriano de Mello, quien en 1783 lenvanta un local comercial y vivienda en la actual calle Zabala de Montevideo. Comprada posteriormente por el Gral. Juan Antonio Lavalleja es hoy sede del Museo Histórico Nacional (Fig. 2).

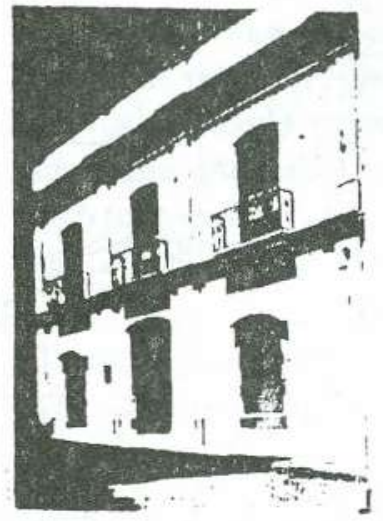

Casa de Juan Antonio Lavalleja Montevideo). Vista exterior.

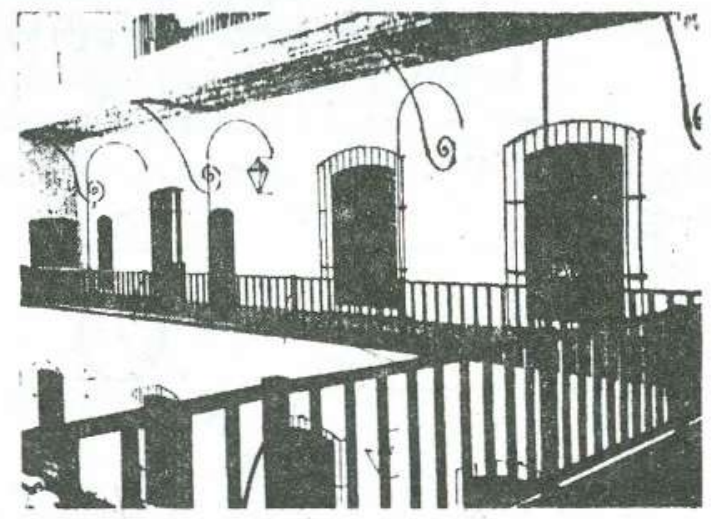

Casa de Juan Antoni Lavalleja (Montevideo). Vista interior.
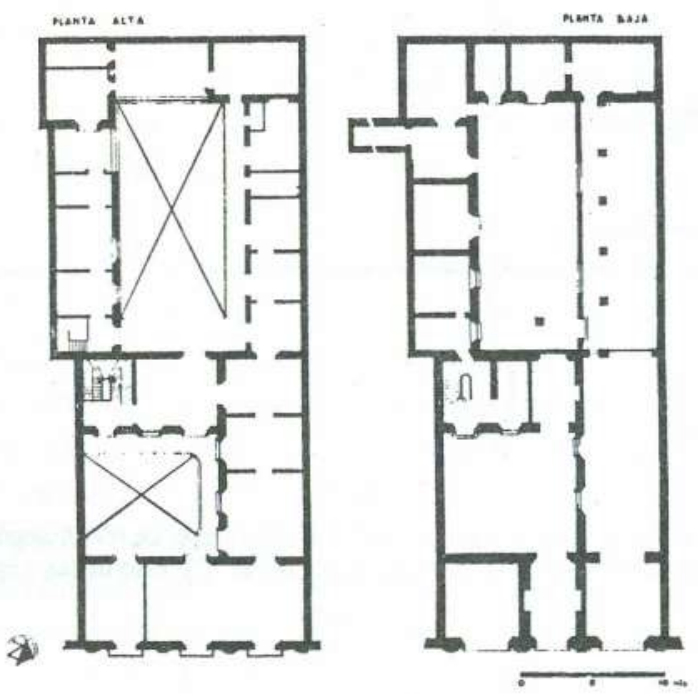

FIG. 2 - Casa de Juan Antonio Lavalleja (Montevideo). Plantas. 


\section{Localización de la población negra en la República}

Gracias a la manumisión y posteriormente al proceso de liberación de la esclavitud en el siglo XIX en nuestron territorio, muchos esclavos dejaron de serlo, ingresando primeramente en la categoría de libertos y luego de hombres libres. Pero siguen pesando sobre ellos un conjunto de limitaciones que son causa de una estructura de clases de la sociedad. A la salida de la esclavitud, un status inferior los conduce a desempeñar los oficios más humildes y peor remunerados y "aunque muchos fueron albañiles, artesanos independientes y soldados de línea, muchos otros tuvieron que desempeñarse como mucamos, aguateros, cocheros". ${ }^{1}$ Una cantidad importante continúa trabajando para sus ex-amos como cocineros, mucamas y lavanderas. Ahora ya no habitan en la vieja residencia patriarcal colonial, deben ubicarse en localizaciones de la ciudad Nueva primero y de la ciudad en extensión después (Fig. 3), en los sectores o barrios del sur y ocupando particulares programas arquitectónicos que la ciudad ofrece a una demanda creciente de inmigrantes extranjeros y de contingentes inmigratorios internos. Los amanzanamientos del sur de la vieja ciudad los nuevos barrios Sur y Palermo principalmente localizan en casas de inquilinato y en conventillos a la población negra de la ciudad.

\section{El conventillo}

El conventillo es una vivienda colectiva, en la cual se arriendan individualmente las habitaciones, siendo de uso común los servicios higiénicos, las cocinas y las piletas de lavar la ropa. Constituye un nuevo programa arquitectónico de habitación colectiva, frente a la casa de inquilinato que resulta de la adaptación de antiguas viviendas unifamiliares, alquiladas por habitación y manteniéndose el servicio higiénico y la cocina original para el uso común.

El sector poblacional que se aloja en los conventillos está constituído mayormente por negros, pero también por inmigrantes y en general por sirvientes y trabajadores informales (lavanderas, costureras, lustrabotas, changadores, mandaderos, etc.). ${ }^{2} \mathrm{El}$ programa conventillo surge como una de las respuestas a la gran demanda de vivienda generada a partir de 1850 , consecuencia a su vez de los cambios de orden político y económico de una sociedad liberal que no da respuesta al problema habitacional a través del Estado. Los sectores de más bajos recursos 


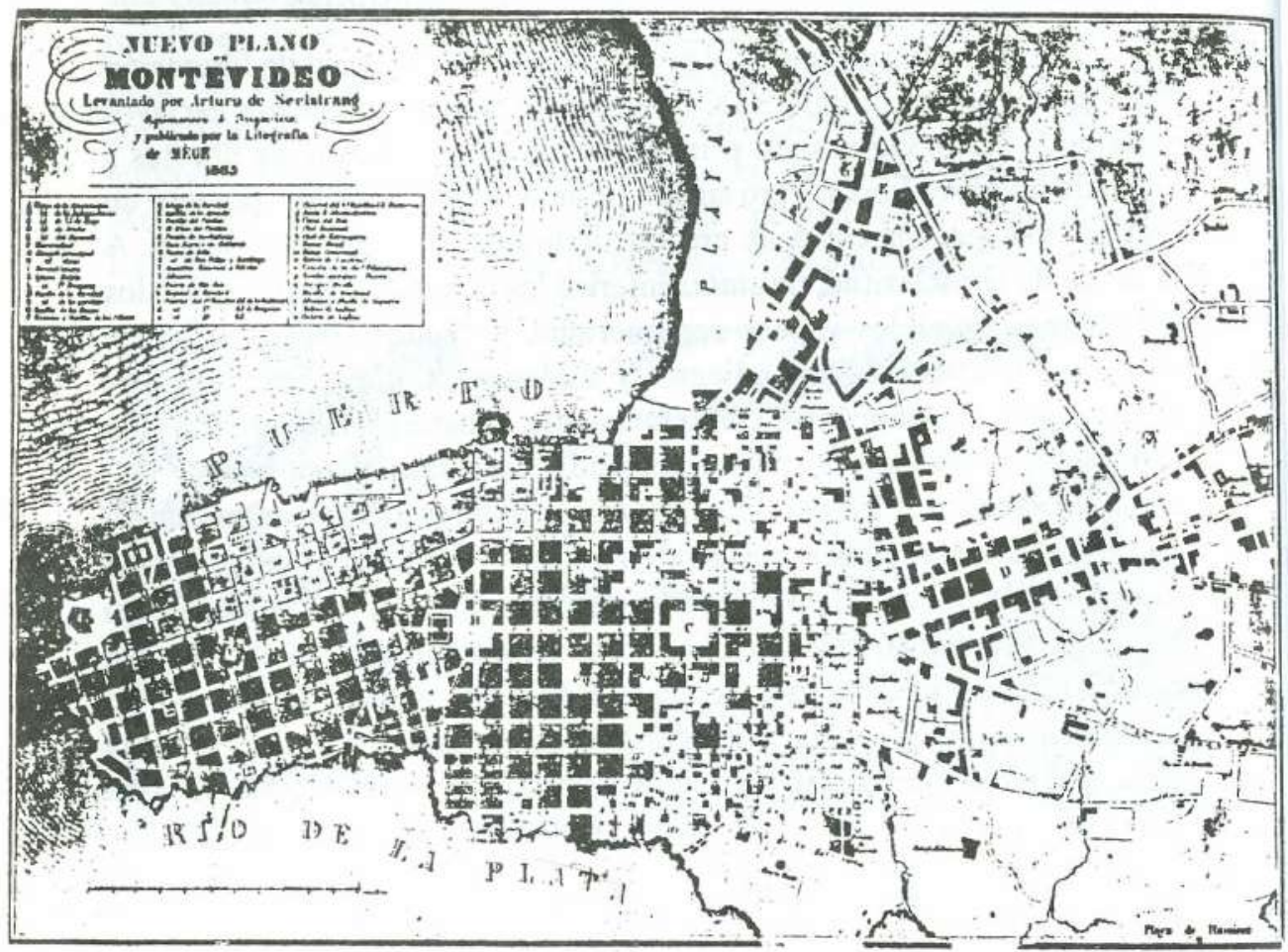

FIG. 3 - Nuevo plano de Montevideo, levantado por el Ing. Arturo de Sellstrang. Añ 1865.

dependerán del inversor privado para solucionar su problema. La iniciativa privada, por su parte, busca obtener una renta sustanciosa a la inversión del capital, sin preocuparse de aspectos humanos o higiénicos. "En estas circunstancias, las condiciones de higiene y habitabilidad de los conventillos son deficientes, registrándose en ellos los más altos índices de morbidad en enfermedades epidémicas (fiebre amarilla, cólera, viruela) y endémicas (tuberculosis)". ${ }^{3}$ Recién en 1871 la Junta Municipal aprueba el "Reglamento de los conventillos de la Capital" y a partir de éste, se suceden las reglamentaciones que exigen mejores condiciones de salubridad e higiene. Sin embargo, una crónica de 1911 nos dice que "muy pocos conventillos poseen los tanques de agua exigidos para los servicios 
higienicos". Octavio Morató nos dice al respecto: "Hemos definido al conventillo con las caracteristicas de la habitación cara y mala. Así como hemos definido al inquilinato como antesala de la muerte, porque no puede pedirse nada peor [...]". ${ }^{4}$ En 1911 se propone la clausura de todas las casas de inquilinato incluidos los conventillos. La ordenanza establece que la Sección de Salubridad proceda a "la clasificación de los edificios de cuartería, a fin de intimar el desalojo en el plazo fijado a sus dueños o encargados". La ordenanza no se cumple y es recién en 1928 que la Ordenanza de Higiene de las Viviendas, finalmente impide la realización de conventillos al establecer los artículos que exigen cocinas y servicios higiénicos "obligatorios para toda casa-habitación". Subsisten sin embargo los conventillos construidos con anterioridad a la mencionada ordenanza, aumentando a su vez las casas de construcción antiguas, convertidas por sus propietarios en inquilinatos. ${ }^{5}$

La ubicación de los conventillos se da en las zonas perimetrales de la ciudad, barrios Sur, Palermo, Cordón y Aguada y Arroyo Seco, proximos a la bahía de Montevideo, evidenciando, por parte de los inversores, la búsqueda de las zonas de más bajo valor económico para la implantación de los conventillos. En cuanto a sus características arquitectónicas generales, podemos reconocer los patios (Fig. 4), sede de reunión de vecinos, lavado y tendedero de ropa a su vez; ubicar los servicios higiénicos comunes así como las cocias, aunque no todos los conventillos la poseían. La ordenanza de proveer a cada habitación de una ventilación en el techo contempla este caso, donde la preparación de alimentos se lleva a cabo en el interior de las habitaciones, aunque en la práctica también se cocina en el patio. También se encuentran conventillos sin servicios higiénicos, aunque los había con más de uno, así como cocinas en número de tres o cuatro como en el conventillo Barouquet. Las piletas de lavar son aprovechadas en los conventillos de las áreas céntricas, para que las morenas laven ropa para sus antiguos amos y otras familias que les pagan por el servicio, a pesar de que esta actividad está prohibida en el reglamento de 1871. El conventillo "Medio Mundo" o de Risso, construido en 1883 en el Barrio Sur (Fig. 5) es uno de los más conocidos popularmente por ser tradicional asiento de población negra y sede de cultura de ésta. Tenía 40 habitaciones, en dos plantas en torno a un patio central con pileas de lavar, con servicios higiénicos comunes pero sin cocinas de uso general. 


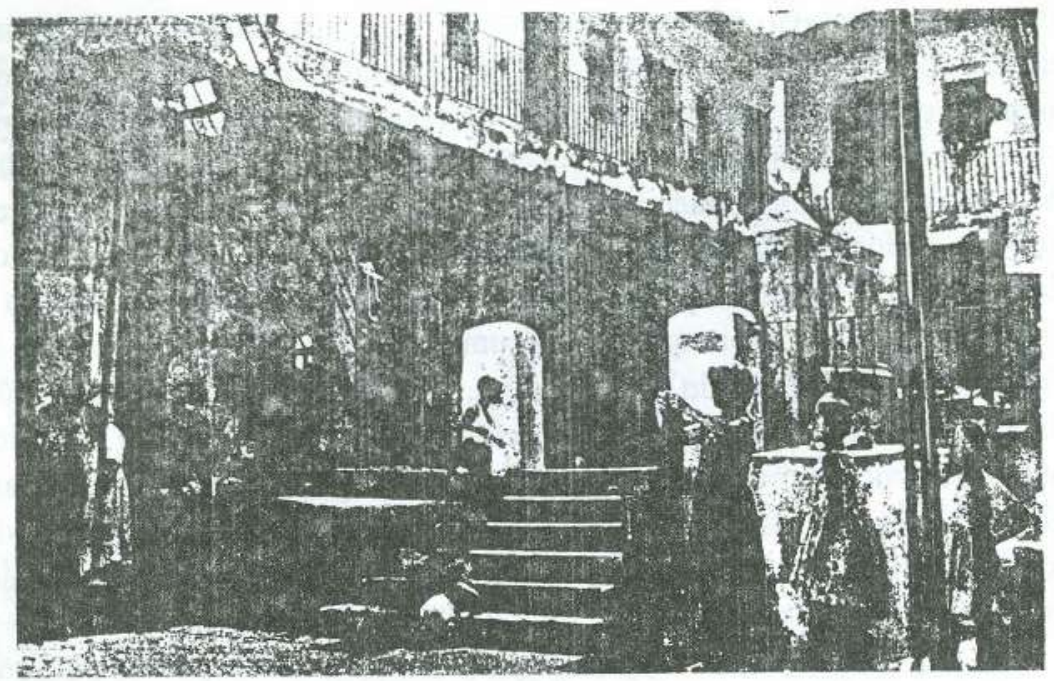

FIG.4 - Patio del conventillo "Medio Mundo".
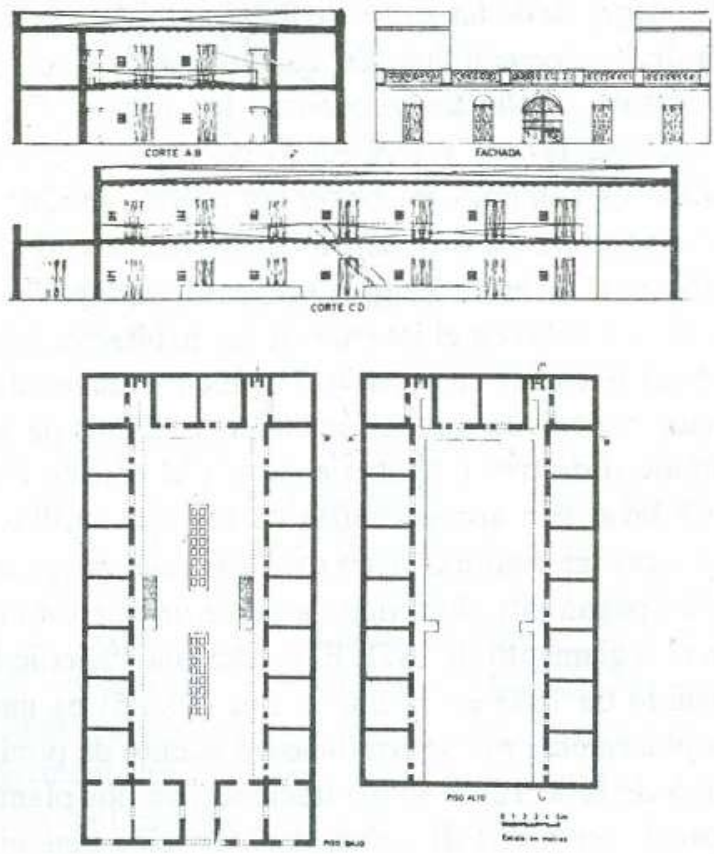

FIG. 5 - Conventillo "Medio Mundo". 
Este particular programa arquitectónico, asiento de la cultura negra, particularmente vinculada al Carnaval negro de la ciudad, contaba con la protección de monumento histórico hasta 1979. En ese año fue desafectado como tal, expulsada su población y demolido. En 1973, con motivo de estar en la consideración pública la venta del edificio, un diario montevideano ${ }^{6}$ atestigua: "Un pedazo de historia del Uruguay y su folklore se vende por 6 millones de pesos. El Conventillo 'Medio Mundo' epicentro de las 'llamadas' fiestas negras que tienen prestigio internacional, como genuina expresión del arte de los morenos orientales corre riesgo de perderse para el acervo histórico-cultural de la ciudad". Seis años más tarde, en vísperas del desalojo de sus moradores, por declaración de "estado ruinoso", se realiza en el conventillo una gran fiesta al son de los tamboriles. La prensa registra: "Una fiesta como sólo puede producir-se en este inigualado 'Medio Mundo', seguramente un fenómeno que no registra nada siquiera parecido en ninguna parte ... acaso la explicación está en la raíz de una raza como la negra, donde las penas parecen arrancar en el fondo de la historia, pero siempre acompañadas del redoble de un tamboril, con el ritmo metido en el cuerpo, casi como una manifestación espiritual de su esencial".?

Las fiestas negras siguen siendo estrellas de nuestro carnaval montevideano y patrimonio de la cultura negra. El predio vacío del "Medio Mundo" asiste año a año a la pasada obligada del desfile de las "llamadas" incorporado a la cultura ciudadana, a la música que generó y a los rituales que la acompañan.

\section{Notas}

1. Pi Hugarte, R, Vidart, D. "El legado de los inmigrantes". Montevideo, 1969.

2. Alvarez Lenzi et alli. "El Montevideo de la expansión". Montevideo, 1986.

3. Morató, O. "Problemas sociales". Montevideo, 1911.

4. Ibidem.

5. Alvarez Lenzi. Ibidem.

6. Diario Acción. Montevideo, 29 de enero de 1973.

7. Diario El Dia. Montevideo, 4 de dic. de 1978. 